\title{
Preparation of Carbon Dots with High-Fluorescence Quantum Yield and Their Application in Dopamine Fluorescence Probe and Cellular Imaging
}

\author{
Haitao Lin, Jun Huang, and Liyun Ding \\ National Engineering Laboratory for Fiber Optic Sensing Technology, Wuhan University of Technology, Wuhan 430070, China \\ Correspondence should be addressed to Liyun Ding; dlyw@whut.edu.cn
}

Received 21 March 2019; Revised 27 May 2019; Accepted 1 July 2019; Published 17 October 2019

Academic Editor: Paulo Cesar Morais

Copyright @ 2019 Haitao Lin et al. This is an open access article distributed under the Creative Commons Attribution License, which permits unrestricted use, distribution, and reproduction in any medium, provided the original work is properly cited.

\begin{abstract}
Carbon dots represent a kind of fluorescent nanomaterial and have broad application prospects in the field of biosensing and optoelectronics. Here, we explored carbon dots with a high-fluorescence quantum yield rate synthesized from L-cysteine and citric acid by the microwave-assisted method. The characteristics of the carbon dots were studied using a transmission electron microscope, an X-ray diffractometer, X-ray photoelectron spectra, a UV-Vis absorption spectrometer, a FTIR spectrometer, and a fluorescence spectrophotometer. The obtained carbon dots exhibited a high-fluorescence quantum yield (up to 85\%), which is due to the combination of amidogens and sulfydryl with carbon dots, and hence bring the improved fluorescence property. We used carbon dots for in vitro imaging of CRL-5822 cells and human umbilical vein endothelial cells, which showed the low inhibitory rate $(0.8 \%)$ of cells for $48 \mathrm{~h}$ with good biocompatibility demonstrated by the cell viability assay. The image of cells can be observed clearly under UV light. The Stern-Volmer equation was introduced to describe the quenching effect between the fluorescence intensity of carbon dots and the concentration of aqueous dopamine (DA).
\end{abstract}

\section{Introduction}

Carbon dots are "zero-dimensional" nanoparticles with carbon structures [1]. As a relatively new member in the big family of fluorescent materials, carbon dots have drawn ever-increasing attention for their promising properties, such as high chemical stability, low blinking, and good biocompatibility $[2,3]$. These characteristics have been exploited for a range of applications including chemical sensing, bioimaging, biolabelling, biomedicine [4], photocatalytic energy conversion [5], and as nanocarriers for gene delivery [6]. Especially for in vivo and in vitro biological purposes, fluorescence probes require not only typical fluorescent properties but also the vitality of host cells or organisms. Therefore, low cytotoxicity, one of the key features of carbon dots, makes them of promising significance in the field of in vivo and in vitro biology. Moreover, the fluorescence wavelength of the carbon dots is dependent on the excitation wavelength in certain ranges, making them more flexible in applications.
The fluorescence behaviors of carbon dots are determined by their sizes, crystalline degrees, and functional groups. Notably, "naked" carbon dots are usually reported to be low in fluorescence quantum yield, but they can be dramatically enhanced by chemical modification or functionalization with organic molecules [5]. As a result, to get carbon dots with a high quantum yield, researchers systematically studied the synthesis of the carbon core to the modification of the surface and proposed the easier concept of "one-stepsynthesis" to harvest high-fluorescence carbon dots from a single step of preparation. Featuring the characteristics of easy methodology, organic solvent-free reaction, economy, efficiency, and security, microwave assistant synthesis is one of the most promising ways towards the preparation of fluorescent carbon dots. The precursor of carbon dots is essential for the fluorescence behavior because some chemical structures of both the core and the surface of the carbon dots originate from the diversified carbon sources and possess high design flexibility, even varying from daily food to beverages, such as coffee [7], green tea [8], pumpkin [9], and potato 
and cucumber [10]. Light-emitting diode, photocatalysis, and fluorescent sensing represent the typical application of carbon dots, among which the study of carbon dots with a high-fluorescence quantum yield plays a vital role. Therefore, the synthesis of carbon dots with strong fluorescence and high-fluorescence quantum yield remains the focus of the carbon dot area.

DA, as a significant biological neurotransmitter, exists extensively in the inside of the living creature's body and participates in a number of motivated behaviors such as brain activity, cardiovascular system activity, and neurotransmission [11]. The abnormal levels of DA concentration in patients are related to many serious diseases especially neurologic diseases, such as Parkinsonism, schizophrenia, Huntington, and Alzheimer [12-15]. Thus, a sensitive and precise detection method of DA is of great importance in clinical determination. Owing to the electroactive nature of DA, many measures based on electrochemical techniques have been proposed. However, due to the existence of many interfering substances and their similar oxidized potentials to DA, the deviations would be taken in the results of DA determination. Therefore, it is still of great necessity to focus on the further development of a facile, convenient, and highly sensitive and selective method for the determination of DA. Compared with the traditional detection method, there are many advantages including stable photoluminescence, highly responsive speed, excellent biocompatibility, good sensitivity, and selectivity for carbon dot-based fluorometric detection. As a promising substitute to the traditional detection method, the fluorescent properties of carbon dots were studied for DA detection.

In this study, carbon dots with high fluorescence were synthesized from L-cysteine and citric acid (both also used in the food industry) by the facile and economic approach of the short-time microwave heating method. The fluorescence properties and cytotoxicity of the prepared carbon dots are studied in detail. Furthermore, the application of carbon dots as a fluorescent probe for in vitro imaging of CRL5822 cells (a typical cancer cell) and human umbilical vein endothelial cells (HUVEC) is also studied. The quenching behavior of carbon dots by the DA solution was found and further demonstrated, and the fluorescence intensity of carbon dots possessed a direct correlation to the concentration of DA in aqueous solutions.

\section{Material and Methods}

2.1. Materials. All chemicals used were of analytical reagent grade without further purification. L-Cysteine (AR), citric acid (AR), and DA hydrochloride (AR) were purchased from Sinopharm Chemical Reagent Co., Ltd. Dulbecco's Modified Eagle's Medium/Nutrient Mixture F-12 (DMEM/F-12), DMEM (high glucose), Minimum Essential Medium/Earle's Balanced Salt Solution (MEM/EBSS), and Roswell Park Memorial Institute-1640 (RPMI-1640) were purchased from HyClone Laboratories Inc. Fetal Bovine Serum (FBS), Trypsin, Thiazolyl Blue Tetrazolium Bromide (MTT), and Dimethyl sulfoxide (DMSO) were purchased from Zhejiang Tianhang Biotechnology Co. Ltd. and AMRESCO Inc., respectively.
The human serum was purchased from ShangHai YuanYe Biotechnology Co., Ltd. Lab-made deionized water was used throughout the experiments.

2.2. Preparation of Carbon Dots. The carbon dots were prepared by microwave-assisted rapid synthesis. $0.57 \mathrm{~g} \mathrm{~L}$-cysteine was dissolved in $10 \mathrm{~mL}$ deionized water under agitation and then mixed with $1.5 \mathrm{~g}$ citric acid. After ultrasonication for 10 mins, the obtained solution was heated for $4 \mathrm{~min}$ in a microwave oven. Following the reaction, the product was cooled down to room temperature naturally and then dissolved in $10 \mathrm{~mL}$ deionized water under agitation to get a brown solution. Finally, it was centrifuged at $9000 \mathrm{rpm}$ for $10 \mathrm{~min}$ to remove the impurity and filtered by a filter. The obtained solution was extracted by ethyl acetate and then kept in a refrigerator under $4^{\circ} \mathrm{C}$ for later use.

2.3. Characterization. High-resolution transmission electron microscopy (HRTEM) was conducted on a JEM-2100F STEM (JEOL, Japan). XRD patterns were obtained from an X-ray diffractometer (D8 ADVANCE, Bruker, Germany). The UV-Vis absorption spectra were taken on a UV-2450 spectrometer (Shimadzu, Japan). The fluorescence spectra were recorded using a fluorescence spectrophotometer (F4500, Hitachi, Japan). FTIR spectra were analyzed using a Fourier transform infrared spectrometer. X-ray photoelectron spectra (XPS) were acquired on an AXIS Ultra DLD X-ray photoelectron spectrometer (Kratos, Tokyo, Japan). Cellular images were captured using an inverted biologic microscope and an inverted fluorescence microscope.

2.4. MTT Assay. CRL-5822 cells and human umbilical vein endothelial cells (HUVEC) were selected for the cell cytotoxicity test. A prepared cell suspension was plated on a Costar ${ }^{\circledR}$ 96-well plate and cultured at $37^{\circ} \mathrm{C}$ with $5 \% \mathrm{CO}_{2}$ for $24 \mathrm{~h}$ to adhere cells onto the surface of the cover glass. Then, the cells that did not undergo treatment with carbon dots were taken as the reference group. The four test groups consisted of carbon dots in aqueous solutions with different concentrations and cells, and then cultured for another $48 \mathrm{~h}, 24 \mathrm{~h}$, and $6 \mathrm{~h}$ respectively. We added $20 \mu \mathrm{L}$ of $5.0 \mathrm{mg} \cdot \mathrm{mL}^{-1}$ MTT solution into each well, and the cells were further incubated for $2 \sim 4 \mathrm{~h}$ to restore MTT to formazan. After $150 \mu \mathrm{L}$ of DMSO was added to remove formazan, the optical density (OD) of the mixture was measured at $490 \mathrm{~nm}$ with an ELIASA. The inhibitory rate $I$ of cells, which was inversely proportional to the cell survival rate, can be calculated according to the following formula [16]:

$$
I(\%)=1-\left(\frac{A_{t}}{A_{C}}\right),
$$

where $I$ depicted the inhibitory rate and $A_{t}$ and $A_{c}$ represent the absorbency of the test group and the control group, respectively.

2.5. Cellular Imaging. The CRL-5822 cells and HUVEC were cultured in DMEM solution, which was supplemented with FBS to prepare a cell suspension. A culture dish with 6 wells containing $2 \mathrm{~mL}$ of cell suspension in each well was used. 
Then, the culture dish was transferred into an incubator for $24 \mathrm{~h}$ to adhere cells onto the surface of carbon dots. After this, further cell treatment was conducted by replacing the medium with serum-free medium for $6 \mathrm{~h}$. The $1: 50$ configuration of the medium with carbon dots was added into hunger-treated cells for $6 \mathrm{~h}$ and $12 \mathrm{~h}$ respectively for optical imaging. Finally, phosphate-buffered solution was used to wash the cells to remove extracellular carbon dots for microscopic analysis.

\section{Results and Discussion}

3.1. The Structures of Carbon Dots. The synthesized carbon dots were well separated in the solution with high fluorescence. The inset in Figure 1 shows that the synthesized carbon dots are near-spherical particles with diameters in the range of 2-4 $\mathrm{nm}$. The HRTEM picture illustrates that the carbon core is well crystallized, with interlattice spacing around $0.31 \mathrm{~nm}$, corresponding to the (002) interplanar distances in the carbon materials with turbostratic disorder and to the shoulder peak at 25 degrees in the XRD pattern of carbon dots as shown in Figure S1 [17].

The FTIR spectra of the prepared carbon dots are shown in Figure S2. The peak in the region of $3036-3600 \mathrm{~cm}^{-1}$ is identified as the stretching vibration absorption peak of the $\mathrm{O}-\mathrm{H}$ and N-H band, and the small band at $2927 \mathrm{~cm}^{-1}$ could be aroused by the stretching vibrations of the $\mathrm{C}-\mathrm{H}$ bond. The relatively weaker characteristic peaks at $2500-2600 \mathrm{~cm}^{-1}$ are consistent with the stretching vibration peaks of $-\mathrm{SH}$. The peak at $1711 \mathrm{~cm}^{-1}$ corresponds to the stretching vibration peak of the $\mathrm{C}=\mathrm{O}$ bond. The peak at $1639 \mathrm{~cm}^{-1}$ is assigned to the stretching vibrations of $\mathrm{C}-\mathrm{O}$ and the bending vibrations of $\mathrm{N}-\mathrm{H}$ [18]. The peak at $1399 \mathrm{~cm}^{-1}$ is related to the stretching vibration of the $\mathrm{C}-\mathrm{N}, \mathrm{N}-\mathrm{H}$, and - $\mathrm{COO}$ bonds. The above observations confirm that reductive groups, such as $-\mathrm{OH},-\mathrm{NH}_{2}$, and $-\mathrm{COO}$, exist on the fringe of carbon dots, which have been reported to be able to improve the fluorescence of carbon dots $[19,20]$. As shown in Figure S3(a), the XPS full scan spectra of carbon dots indicate that there are four clear peaks around $163.17 \mathrm{eV}, 284.64 \mathrm{eV}, 400 \mathrm{eV}$, and $531.69 \mathrm{eV}$ ascribed to sulfur $(4.42 \%)$, carbon $(57.97 \%)$, nitrogen $(5.46 \%)$, and oxygen $(32.15 \%)$, respectively [21]. The spectrum of $\mathrm{N} 1 \mathrm{~s}$ shown in Figure S3(b) includes peaks at $400 \mathrm{eV}$ for C-N, which indicated that the chemical groups $-\mathrm{NH}_{2}$ immobilized on the carbon dots had only one state (C$\mathrm{NH}_{2}$ ) [2]. In Figure $\mathrm{S} 3(\mathrm{c})$, the $\mathrm{C}=\mathrm{O}$ and $\mathrm{O}-\mathrm{H}$ units corresponded to peaks at $531.6 \mathrm{eV}$ and $533.1 \mathrm{eV}$, respectively [22]. From Figure S3(d), it is shown that the C 1s peaks at $284.4 \mathrm{eV}, 286.2 \mathrm{eV}$, and $288.3 \mathrm{eV}$ corresponded to $\mathrm{C}-\mathrm{C}, \mathrm{C}-\mathrm{O} / \mathrm{C}-\mathrm{N}$, and $\mathrm{C}=\mathrm{O}$, respectively [2]. These results were in consonance with those of FTIR spectometry, demonstrating that the common hydrophilic groups such as amidogen, carboxylic, and hydroxyl from the precursor were immobilized on the edge of the synthesized carbon dots. These can make contributions to the improvement of the solubility of carbon dots, which improves the dispersion of carbon dots in an aqueous solution to obtain a homogeneous carbon quantum dot solution and reduces

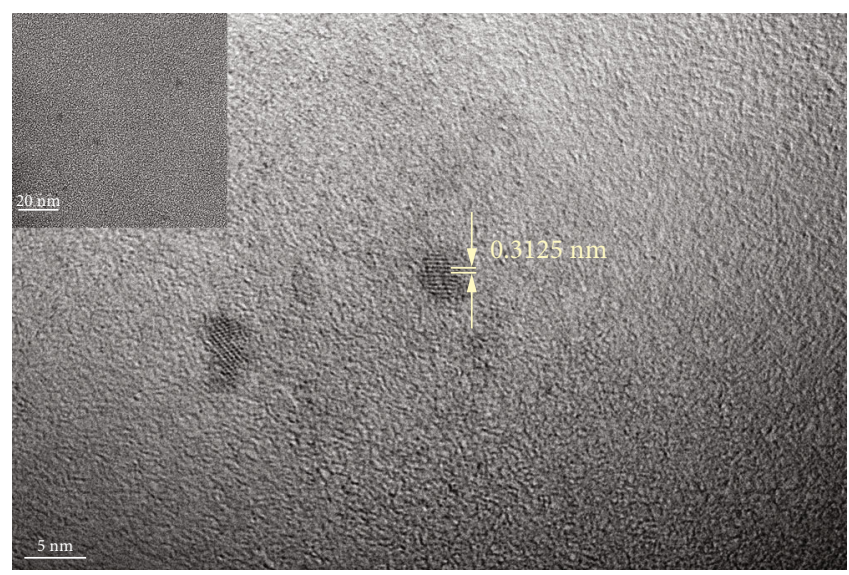

FIgURE 1: The high-resolution transmission electron microscopy images of a single carbon dot.

the loss of a quantum confinement effect of carbon dots due to agglomeration. As a result, the fluorescence property of carbon dots was improved.

3.2. The Optical Properties of Carbon Dots. The UV-Vis absorption spectrum and fluorescence spectra (excitation wavelength $=355 \mathrm{~nm}$ ) of carbon dots are shown in Figure 2 . The carbon dot solution (with pictures in the upper right corner of Figure 2) changes from colorless to bright blue after excitation by UV light. The absorption peak at $341 \mathrm{~nm}$ originates from the $n-\pi *$ transition of $\mathrm{C}=\mathrm{O}$ bonds, which is similar to the results of other published works [23-25]. Based on the consistent shape of absorption spectra and fluorescence excitation spectra, in the near UVVis range, the carbon dots' optimal excitation wavelength is in the vicinity of $340 \mathrm{~nm} \sim 360 \mathrm{~nm}$, resulting in fluorescence at $\sim 439 \mathrm{~nm}$. Figure S4(a) shows that under excitation with a different wavelength, the fluorescence emission not only varies in intensity but also shifts in wavelength, known as excitation-dependent fluorescence emitting. The normalized fluorescence spectra of carbon dots are displayed in Figure S4(b), clearly demonstrating that the emitting red shifted from $435 \mathrm{~nm}$ to $460 \mathrm{~nm}$ while the excitation wavelength increased from $300 \mathrm{~nm}$ to $400 \mathrm{~nm}$. This phenomenon can be explained by the nonuniform size distribution of carbon cores and the multiple surface sites existing on carbon dots. The nonuniform size of the carbon cores and the multiple surface sites which had different optimum excitation wavelengths emitted different optimum fluorescence levels at various excitation wavelengths. As a result, the emission wavelength of carbon dots varies with the excitation wavelength. Quinine sulfate with a fluorescence quantum yield of 0.54 was chosen as the standard fluorescent agent because of the similar excitation and emission wavelength with the prepared carbon dots. The quantum yield with reference is calculated by the following equation [26]:

$$
\varphi_{\mathrm{x}}=\varphi_{\mathrm{st}}\left(\frac{K_{\mathrm{x}}}{K_{\mathrm{st}}}\right)\left(\frac{\eta_{\mathrm{x}}}{\eta_{\mathrm{st}}}\right)^{2}
$$




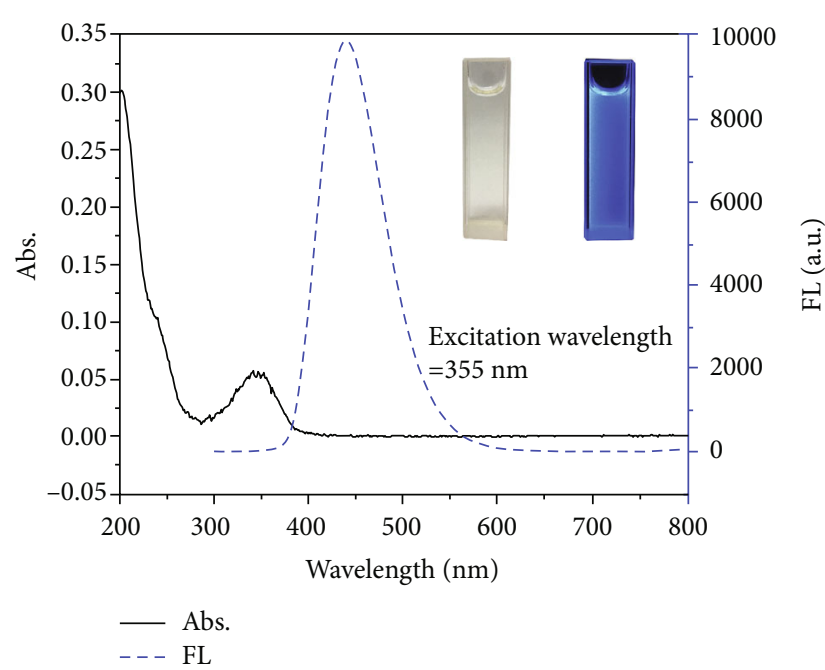

FIGURE 2: UV-Vis absorption spectrum and fluorescence spectrum of carbon dots. The right inset photographs show carbon dots exposed to daylight and UV light, respectively.

where $\varphi, K$, and $\eta$ are the quantum yield, gradient from the linear regression, and refractive index of solution, respectively. The subscripts "st" and " $\mathrm{x}$ " refer to the standard fluorescent agent and the tested sample, and the value of $\eta_{\mathrm{x}} / \eta_{\mathrm{st}}$ is 1 for those two solutions with extremely low concentrations. The absorbances of both the sample and standard fluorescent agent were kept under 0.1 to guarantee a linear relationship between the absorbance and the molar concentration. Finally, the fluorescence quantum yield of carbon dots is $85 \%$ (shown in Figure S5), given by the slope of the diagram of fluorescence integral intensity to the absorbance. The chemical groups of $-\mathrm{NH}_{2}$ contributed to the fluorescence variation of carbon dots and improved their fluorescence quantum yield, which could be explained by the edge-treatment-dominated optical tunability caused by the resonance between the $\pi$ orbital and the molecular orbital in the $-\mathrm{NH}_{2}$ groups in $\mathrm{N}$ doped carbon dots $[19,20]$. The surface passivation of carbon dots with organic groups (especially $-\mathrm{NH}_{2}$ groups) can improve the emission fluorescence [27]. This was demonstrated by the experimental observation of the high quantum yield of carbon dots. In previous reports, the sulfur and nitrogen dopant strengthened the catalytic ability for carbon materials [28-30]. It seems that the $-\mathrm{SH}$ groups would improve the above fluorescence-enhancing effect of $-\mathrm{NH}_{2}$ groups on the performance of the doped carbon nanomaterials through a synergistic effect [31]. The chemical group of $-\mathrm{SH}$ and $-\mathrm{NH}_{2}$ were combined with the carbon dots synthesized by microwave simultaneously. The high degree of recrystallization of carbon dots with the inside-out heating method and the sulfur and nitrogen groups contribute to the high-fluorescence quantum yield of these carbon dots. The quantum yield value remains up to $81 \%$ even after extraction by ethyl acetate, which still exceeded the recent report value [32]. Table 1 shows the quantum yield of carbon dots in previous works. The quantum yield of carbon dots which was functionalized
TABLE 1: The quantum yield of carbon dots in previous reports.

\begin{tabular}{lcc}
\hline Types of carbon dots & Quantum yield & Ref. \\
\hline Carbon dots with amino groups & $7.8 \%$ & {$[18]$} \\
$\begin{array}{l}\text { Carbon dots passivated by oligomeric } \\
\text { poly(ethylene glycol) diamine }\end{array}$ & $20 \%$ & {$[33,34]$} \\
$\begin{array}{l}\text { Carbon dots with the surface } \\
\text { passivation processing }\end{array}$ & $50 \%$ & {$[33,34]$} \\
$\mathrm{CZnS}$ dots & $78 \%$ & {$[35]$} \\
$\mathrm{CTiO}_{2}$ dots & $70 \%$ & {$[35]$} \\
$\mathrm{Nitrogen}^{-d o p e d ~ c a r b o n ~ d o t s ~}$ & $46.2 \%$ & {$[36]$} \\
Sulfur-doped carbon dots & $58 \%$ & {$[37]$} \\
Gadolinium-doped carbon dots & $69.86 \%$ & {$[38]$} \\
Carbon dots & $81 \%-85 \%$ & This work \\
\hline
\end{tabular}

with amino groups through the hydrothermal carbonization of chitosan by Yang et al. is about 7.8\% [18]. The surface of carbon dots was passivated by oligomeric poly(ethylene glycol) diamine (PEG1500N) molecules through the reflux method, and only a $20 \%$ quantum yield was obtained. After further processing of better surface passivation, the quantum yield of carbon dots is higher than $50 \%$ by Wang et al. and Yang et al. [33, 34]. More recently, small carbon dots which were obtained from the laser ablation method were functionalized by a combination of doping with nanoscale semiconductors and organic functionalization, followed by gel column fractionation to harvest the most fluorescent carbon dots. The observed emission quantum yields for $\mathrm{CZnS}$ dots and $\mathrm{CTiO}_{2}$ dots were up to $78 \%$ and $70 \%$, respectively [35]. A kind of nitrogen-doped carbon dot with the utmost quantum yield (46.2\%) was generated through a three-electrode EC system by Niu et al. for photoluminescent and electrochemiluminescent applications [36]. The sulfur-doped carbon dots with a high quantum yield (58\%) synthesized via a microwave-assisted pyrolysis method for ultrasensitive detection of DA was reported by Gupta and Nandi [37]. Gadolinium-doped carbon dots prepared by $\mathrm{Yu}$ et al. via a hydrothermal treatment exhibit enhanced fluorescence and high quantum yield of up to $69.86 \%$ [38]. The surface passivation and the synthetic method of carbon dots were significant factors that affected the quantum yield of carbon dots. We obtained the carbon dots with a high-fluorescence quantum yield by using proper quantities of the chemical group of $-\mathrm{SH}$ and $-\mathrm{NH}_{2}$ codoped carbon dots synthesized via microwave-assisted carbonization which can have greater uniformity of carbonization than other methods. The synergistic effect of the chemical group of $-\mathrm{SH}$ and $-\mathrm{NH}_{2}$ and the uniformity of carbonization played an important role in increasing the quantum yield of carbon dots.

3.3. Carbon Dots as a Fluorescent Agent for Cell Imaging. In order to study the bioimaging accessibility, the capability and performance of carbon dots for cell imaging had also been demonstrated. The prepared carbon dot solution with $0.1252 \mathrm{~g} \cdot \mathrm{mL}^{-1}$ ( $1: 1500$ dilution) was delivered into the sterile culture medium. CRL-5822 cells and HUVEC were processed in accordance with the method in Section 2.5. The 


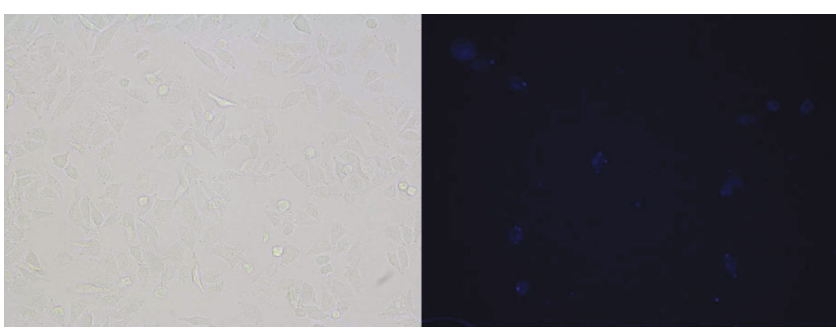

(a)

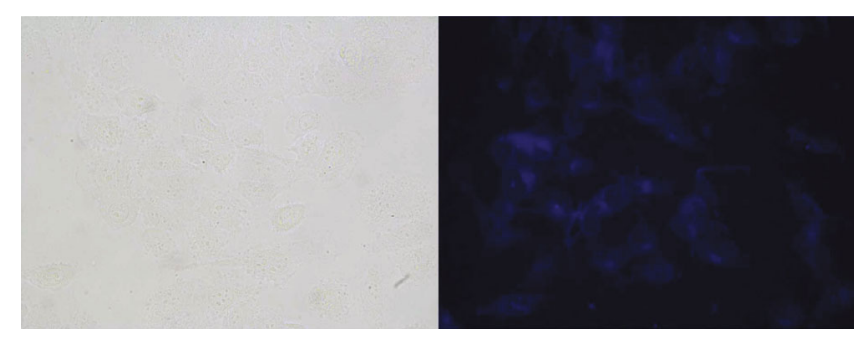

(b)

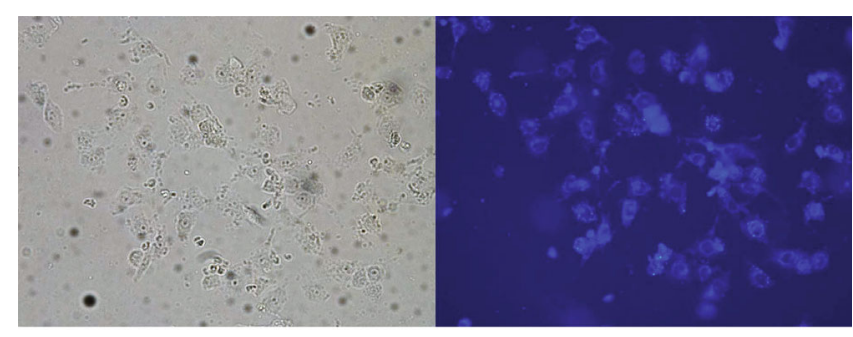

(c)

FIGURE 3: (a) Cellular images of CRL-5822 cells and (b) HUVEC taken in the presence of carbon dots for $6 \mathrm{~h}$ under a bright field and a fluorescent field. (c) Cellular images of HUVEC treated with carbon dots for $12 \mathrm{~h}$ under a bright field and a fluorescent field.

cover glass fully attached with CRL-5822 cells and HUVEC were transferred into the above-treated culture medium to culture the cells, respectively. The carbon dots entered the cells by penetrating the cell membrane and accumulated within the intracellular space including the cytoplasm and the nuclei, which can be observed under the fluorescent microscope as shown in Figure 3. In Figure 3(a), the representative bright-field and fluorescence images of CRL-5822 cells were treated with carbon dots for $6 \mathrm{~h}$. Figure 3(b) shows cell images of HUVEC treated with carbon dots for $6 \mathrm{~h}$. The fluorescence of the HUVEC labeled by carbon dots was stronger than that of the CRL-5822 cells. The bioimage for $6 \mathrm{~h}$ shows that the prepared carbon dots could be accepted by normal cells (HUVEC) more easily than cancer cells. Consequently, this reagent as a fluorescent probe could enter and label the cell quickly. As shown in Figure 3, the prepared carbon dots were internalized into two kinds of cells gradually. Compared with the cells treated by carbon dots for $6 \mathrm{~h}$ in Figures 3(b) and 3(a), the stronger blue fluorescence of the prepared carbon dots can be evidently observed in the cell membrane and cytoplasm when the cells were treated with carbon dots for $12 \mathrm{~h}$ in Figure 3(c). More importantly, the cell morphology remained unchanged during the whole process, indicating that carbon dots can inflict minimal harm to the cell. Therefore, the enrichment of carbon dots in in vivo cells has hardly any effect on cell viability, that is, the cells treated with carbon dots for a long time still possess enough vitality and proliferating ability. It demonstrated that the synthetic carbon dots possess excellent biocompatibility and a highfluorescence quantum yield, confirming the application potential of carbon dots in biological labelling and imaging.

3.4. Biocompatibility of Carbon Dots. In order to explore the biocompatibility of carbon dots for cell-imaging agents in living cells, CRL-5822 cells and HUVEC were exposed to car- bon dot aqueous solutions for $6 \mathrm{~h}, 24 \mathrm{~h}$, and $48 \mathrm{~h}$, respectively. It is shown that after treating with carbon dot aqueous solutions, the inhibitory rates (the number of survived cells compared with the control group) of two kinds of cells are both high with more concentrated carbon dots. Comparing Figure 4(a) with Figure 4(b), CRL-5822 cells and HUVEC had almost the same inhibitory rate in the first $6 \mathrm{~h}$. After treating for $24 \mathrm{~h}$ and $48 \mathrm{~h}$, carbon dots have a slight influence on the cell viability of HUVEC. As shown in Figures 4(a) and 4 (b), the cells treated for $48 \mathrm{~h}$ have a lower inhibitory rate than others when the concentration of carbon dots is $0.07825 \mathrm{mg} \cdot \mathrm{mL}^{-1}(1: 1600$ dilution). Notably, in some tests with a low concentration of carbon dots, the cell growth was even boosted, suggesting the possible existence of some nutrients which are friendly to either or both kinds of cells. Hence, the prepared carbon dots have great biocompatibility for in vitro and in vivo application.

3.5. DA Detection. When added with DA solution, the fluorescence intensity of carbon dots attenuates accordingly, which means that DA has a powerful ability to quench the fluorescence of carbon dots, and the corresponding quenching effect is shown in Figure 5(a). Due to the existence of chemical groups on the surface of carbon dots, such as $\mathrm{C}=\mathrm{O}$ and $-\mathrm{NH}_{2}$, the carbon dots possess strong fluorescence and higher quantum yield. However, when the DA solution was dropped into the diluted carbon dot solution, the reaction between the N-H bonds of carbon dots and DA structure occurred, in which the DA structure acted as an electron acceptor. This can be explained by the electronic transfer between the carbon dots and DA, causing the fluorescence quenching of carbon dots. Therefore, the fluorescence quenching mechanism among DA molecular and carbon dots can be used to detect the concentration of a DA solution. The Stern-Volmer equation was introduced to precisely 


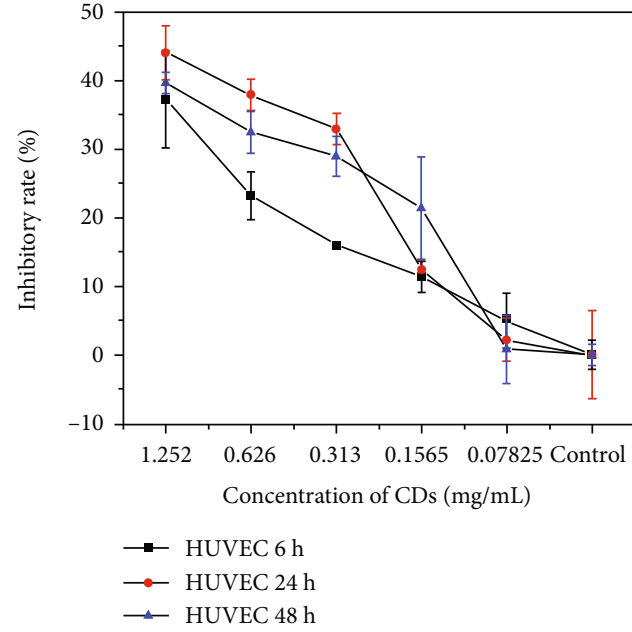

(a)

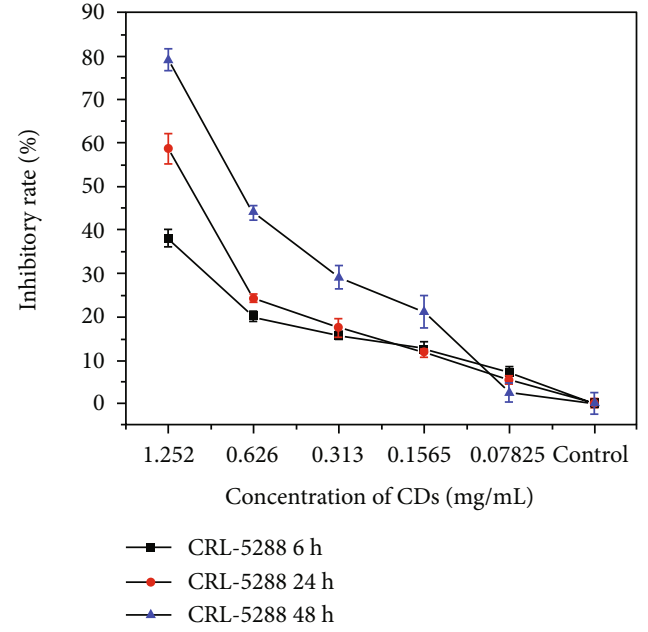

(b)

FIGURE 4: Effect of carbon dots on the cell inhibitory rate of (a) HUVEC and (b) CRL-5822 cells.

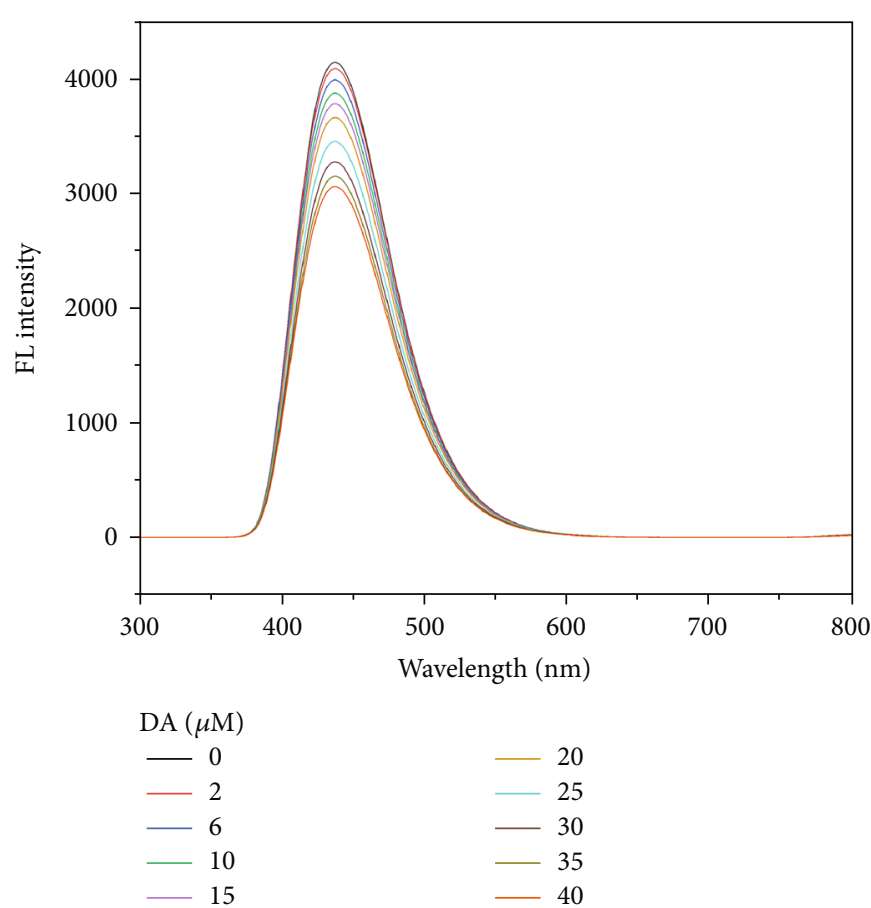

(a)

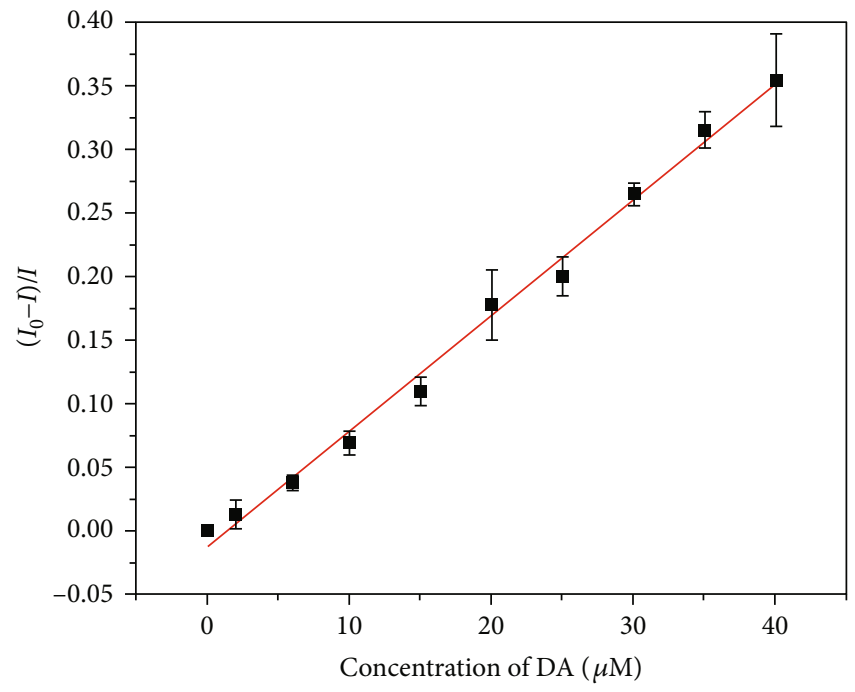

(b)

FIGURE 5: (a) Fluorescence spectrum of carbon dots when adding aqueous DA of different concentrations. (b) The standard fluorescence intensity curve of carbon dots under different DA concentrations.

describe the degree of fluorescence quenching with the changing concentration of aqueous DA [39]:

$$
\frac{I_{0}}{I}=1+K_{\mathrm{sv}}[C],
$$

where $I$ is the fluorescence intensity, $K_{\mathrm{sv}}$ is the slope of the linearity which stands for the constant of quenching, and the concentration of DA is described by $[C]$. The subscript
0 represents the carbon dot solution without the aqueous DA. As shown in Figure 5(b), the linearity $I_{0} / I=1+$ $0.00868[C]\left(R^{2}=0.995\right)$ was achieved in the concentration range from 0 to $40 \mu \mathrm{M}$ of $\mathrm{DA}$ and the limit of detection (LOD) is $0.75 \mu \mathrm{M}(S / N=3)$, which is comparable with previous reports in Table 2. It indicated that the prepared carbon dots with a high quantum yield was highly sensitive to the DA solution, which can be used as a fluorescence probe for DA detection. 
TABLE 2: Comparison of different methods for DA detection.

\begin{tabular}{|c|c|c|c|}
\hline Detection probe & $\begin{array}{c}\text { Linear } \\
\text { range }(\mu \mathrm{M})\end{array}$ & $\begin{array}{l}\text { LOD } \\
(\mu \mathrm{M})\end{array}$ & Ref. \\
\hline ZnO@Cys & $26.3-68.5$ & 0.791 & {$[40]$} \\
\hline MWCNT/GONR & $0.15-12.12$ & 0.08 & {$[41]$} \\
\hline AuNP/PAN electrodes & $1-100$ & 0.91 & {$[42]$} \\
\hline $\mathrm{Fe}_{3} \mathrm{O}_{4} @ \mathrm{SiO}_{2}-\mathrm{NH}-\mathrm{EDTA}-\mathrm{T}_{\mathrm{b}}(\mathrm{III})$ & $2-20$ & 0.82 & {$[43]$} \\
\hline N-GQDs & $0.01-3$ & 0.0033 & {$[44]$} \\
\hline GQDs & $0.25-50$ & 0.09 & {$[45]$} \\
\hline g- $\mathrm{C}_{3} \mathrm{~N}_{4}$ nanofibers & $0-20$ & 0.017 & {$[46]$} \\
\hline r-QDs@SiO 2 -PDA & $10-80$ & 0.12 & {$[47]$} \\
\hline TGA-CdS QDs & $1.0-17.5$ & 0.68 & {$[48]$} \\
\hline Carbon dots & $0-40$ & 0.75 & This work \\
\hline
\end{tabular}

3.6. Selectivity. In order to accurately measure the DA concentration under different environments, the crossinfluence raised by an interfering substance must be eliminated. The interference effect of other chemicals for carbon dots was also studied. In Figure 6, compared with the common chemicals such as cysteine, glycine, ascorbic acid, urea, and glucose, the quenching effect of carbon dots by DA was more obvious, which demonstrated that the detection of DA by carbon dots possessed a distinct specificity based on the fluorescence quenching principle.

3.7. Real Application. In order to evaluate the feasibility of the application of the fluorescent probe in real sample detection, the standard addition method was used in the DA detection based on carbon dots with human serum samples. The results are presented in Table 3 . The satisfictory recovery rates are between $97.70 \%$ and $103.30 \%$ with the relative standard deviation (RSD) in the range from $2.13 \%$ to $2.91 \%$. It can be demonstrated that the fluorescent probe based on carbon dots possesses a good feasibility, acceptability, and accuracy in DA detection with real samples. Thus, the proposed method exhibited the application potential for DA detection in biological samples.

3.8. Conclusions. In conclusion, we have synthesized carbon dots with a high-fluorescence quantum yield (up to $85 \%$ ) by an accessible, rapid, economical, and ecofriendly microwave-assisted approach. To the best of our knowledge, it is higher than any other reported quantum yield value of carbon dots. In our synthesis system, the $-\mathrm{NH}_{2}$ and $-\mathrm{SH}$ are effective chemical groups to increase the fluorescent quantum yield of carbon dots, which proves to be an effective method to enhance the fluorescence of carbon dots. The carbon dots had an excellent luminescence property and good water solubility, as well as fantastic biocompatibility. The best dilution ratio of $1: 1600\left(0.07825 \mathrm{mg} \cdot \mathrm{mL}^{-1}\right.$ concentration) was confirmed. In this ratio, the carbon dots could emit strong fluorescence by excitation and had high biological compatibility. Moreover, the fluorescence images of the CRL-5822 cells and HUVEC exhibited that carbon dots could be efficiently absorbed by living cells. The fine linearity between DA concentration and fluorescence intensity of car-

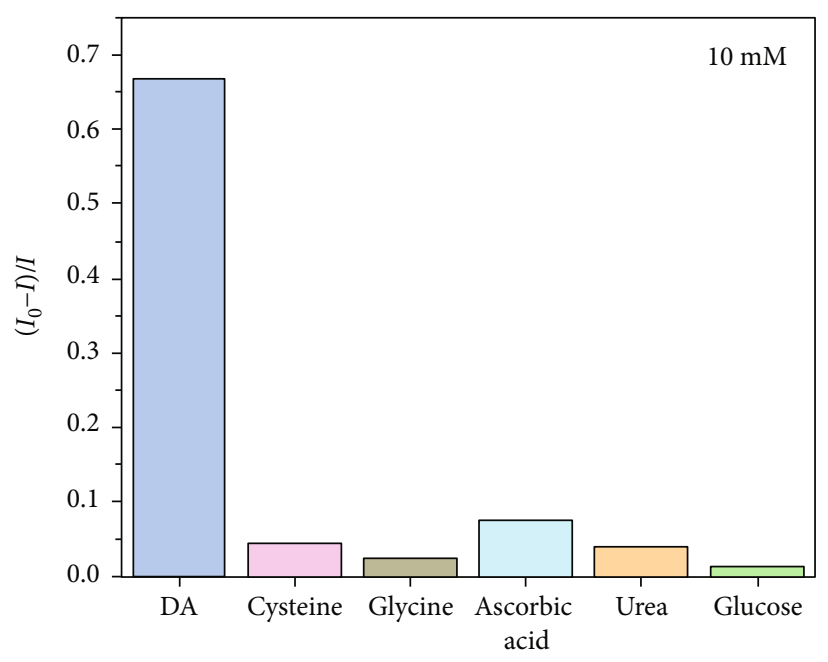

FIGURE 6: Fluorescence response of the carbon dots of $10 \mathrm{mM}$ DA or other an alytes.

TABLE 3: Detection of DA in human serum samples.

\begin{tabular}{lcccc}
\hline Sample & $\begin{array}{c}\text { DA added } \\
(\mu \mathrm{M})\end{array}$ & $\begin{array}{c}\text { DA found } \\
(\mu \mathrm{M})\end{array}$ & $\begin{array}{c}\text { Recovery } \\
\text { rate }(\%)\end{array}$ & $\begin{array}{c}\text { RSD } \\
(n=3, \%)\end{array}$ \\
\hline 1 & 10 & $9.77 \pm 0.21$ & 97.70 & 2.13 \\
2 & 15 & $14.77 \pm 0.48$ & 98.47 & 3.22 \\
3 & 20 & $20.66 \pm 0.60$ & 103.30 & 2.91 \\
\hline
\end{tabular}

bon dots $\left(R^{2}=0.995\right)$ was obtained, which demonstrated that the concentration of the DA solution can be measured reasonably. The cross-influence raised by several common interfering substances and applications in real samples were estimated in detail, demonstrating that the carbon dots had a good selective recognition ability and can be applied for DA detection in biological samples. These features prove that the carbon dots can act as potential candidates for bioimaging and biosensing with few interference and high efficiency.

\section{Data Availability}

The data used to support the findings of this study are available from the corresponding author upon request.

\section{Conflicts of Interest}

There are no conflicts to declare.

\section{Acknowledgments}

This work was financially supported by the National Natural Science Foundation of China (Nos. 61575150 and 51878524)

\section{Supplementary Materials}

Figure S1: the XRD pattern of carbon dots. Figure S2: FTIR spectrum of carbon dots. Figure S3: (a) XPS spectrum, (b) $\mathrm{N}$ 1s spectrum, (c) O 1s spectrum, and (d) C 1s spectrum of carbon dots. Figure S4: (a) the fluorescence spectra of 
carbon dots excited at variant wavelengths and (b) the normalized fluorescence spectra of carbon dots. Figure S5: the relation between fluorescence integral intensity and absorbance (the slope of lines indicated the quantum yield of carbon dots). Quinine sulfate (QS) was selected as a standard indicator. (Supplementary Materials)

\section{References}

[1] S. Y. Lim, W. Shen, and Z. Q. Gao, "Carbon quantum dots and their applications," Chemical Society Review, vol. 44, no. 1, pp. 362-381, 2015.

[2] W. Li, Z. H. Zhang, B. Kong et al., "Simple and green synthesis of nitrogen-doped photoluminescent carbonaceous nanospheres for bioimaging," Angewandte Chemie-International Edition, vol. 52, no. 31, pp. 8151-8155, 2013.

[3] W. J. Niu, Y. Li, R. H. Zhu, D. Shan, Y. R. Fan, and X. J. Zhang, "Ethylenediamine-assisted hydrothermal synthesis of nitrogendoped carbon quantum dots as fluorescent probes for sensitive biosensing and bioimaging," Sensors and Actuators B-Chemical, vol. 218, pp. 229-236, 2015.

[4] P. G. Luo, S. Sahu, S. T. Yang, S. K. Sonkar, and Y. P. Sun, "Carbon "quantum" dots for optical bioimaging," Journal of Materials Chemistry B, vol. 1, no. 16, pp. 2116-2127, 2013.

[5] K. A. S. Fernando, S. Sahu, Y. Liu et al., "Carbon quantum dots and applications in photocatalytic energy conversion," ACS Applied Materials \& Interfaces, vol. 7, no. 16, pp. 8363-8376, 2015.

[6] C. J. Liu, P. Zhang, X. Y. Zhai et al., "Nano-carrier for gene delivery and bioimaging based on carbon dots with PEIpassivation enhanced fluorescence," Biomaterials, vol. 33, no. 13, pp. 3604-3613, 2012.

[7] C. K. Jiang, H. Wu, X. J. Song, X. J. Ma, J. H. Wang, and M. Q. Tan, "Presence of photoluminescent carbon dots in Nescafe ${ }^{\circledR}$ original instant coffee: applications to bioimaging," Talanta, vol. 127, pp. 68-74, 2014.

[8] P. C. Hsu, P. C. Chen, C. M. Ou, H. Y. Chang, and H. T. Chang, "Extremely high inhibition activity of photoluminescent carbon nanodots toward cancer cells," Journal of Materials Chemistry B, vol. 1, no. 13, pp. 1774-1781, 2013.

[9] S. J. Zhao, M. H. Lan, X. Y. Zhu et al., "Green synthesis of bifunctional fluorescent carbon dots from garlic for cellular imaging and free radical scavenging," ACS Applied Materials \& Interfaces, vol. 7, no. 31, pp. 17054-17060, 2015.

[10] C. F. Wang, D. Sun, K. L. Zhuo, H. C. Zhang, and J. J. Wang, "Simple and green synthesis of nitrogen-, sulfur-, and phosphorus-co-doped carbon dots with tunable luminescence properties and sensing application," RSC Advances, vol. 4, no. 96, pp. 54060-54065, 2014.

[11] R. M. Wightman, L. J. May, and A. C. Michael, "Detection of dopamine dynamics in the brain," Analytical Chemistry, vol. 60, no. 13, pp. 769A-779A, 1988.

[12] L. J. Tang, S. Li, F. Han et al., "Sers-active Au@Ag nanorod dimers for ultrasensitive dopamine detection," Biosensors \& Bioelectronics, vol. 71, pp. 7-12, 2015.

[13] Y. Tao, Y. H. Lin, J. S. Ren, and X. G. Qu, “A dual fluorometric and colorimetric sensor for dopamine based on BSA-stabilized Au nanoclusters," Biosensors \& Bioelectronics, vol. 42, pp. 4146, 2013.

[14] T. S. Tang, X. Chen, J. Liu, and I. Bezprozvanny, “Dopaminergic signaling and striatal neurodegeneration in Huntington's disease," Journal of Neuroscience, vol. 27, no. 30, pp. 78997910, 2007.

[15] G. Koch, F. D. Lorenzo, S. Bonni et al., "Dopaminergic modulation of cortical plasticity in Alzheimer's disease patients," Neuropsychopharmacology, vol. 39, no. 11, pp. 2654-2661, 2014.

[16] M. Wu, J. J. Chen, H. Veroniaina et al., "Pea-like nanocabins enable autonomous cruise and step-by-step drug pushing for deep tumor inhibition," Nanomedicine : Nanotechnology, Biology, and Medicine, vol. 18, pp. 122-134, 2019.

[17] H. Peng and J. Travas-Sejdic, "Simple aqueous solution route to luminescent carbogenic dots from carbohydrates," Chemistry of Materials, vol. 21, no. 23, pp. 5563-5565, 2009.

[18] Y. H. Yang, J. H. Cui, M. T. Zheng et al., "One-step synthesis of amino-functionalized fluorescent carbon nanoparticles by hydrothermal carbonization of chitosan," Chemical Communications, vol. 48, no. 3, pp. 380-382, 2012.

[19] H. Tetsuka, R. Asahi, A. Nagoya et al., "Optically tunable amino-functionalized graphene quantum dots," Advanced Materials, vol. 24, no. 39, pp. 5333-5338, 2012.

[20] S. H. Jin, D. H. Kim, G. H. Jun, S. H. Hong, and S. Jeon, “Tuning the photoluminescence of graphene quantum dots through the charge transfer effect of functional groups," ACS Nano, vol. 7, no. 2, pp. 1239-1245, 2013.

[21] D. Sun, R. Ban, P. H. Zhang, G. H. Wu, J. R. Zhang, and J. J. $\mathrm{Zhu}$, "Hair fiber as a precursor for synthesizing of sulfurand nitrogen-co-doped carbon dots with tunable luminescence properties," Carbon, vol. 64, pp. 424-434, 2013.

[22] S. K. Bhunia, L. Zeiri, J. Manna, S. Nandi, and R. Jelinek, "Carbon-dot/silver-nanoparticle flexible SERS-active films," ACS Applied Materials \& Interfaces, vol. 8, no. 38, pp. 2563725643, 2016.

[23] S. J. Zhu, Y. B. Song, X. H. Zhao, J. R. Shao, J. H. Zhang, and B. Yang, "The photoluminescence mechanism in carbon dots (graphene quantum dots, carbon nanodots, and polymer dots): current state and future perspective," Nano Research, vol. 8, no. 2, pp. 355-381, 2015.

[24] Z. T. Luo, Y. Lu, L. A. Somers, and A. T. C. Johnson, "High yield preparation of macroscopic graphene oxide membranes," Journal of the American Chemical Society, vol. 131, no. 3, p. 898, 2009.

[25] Y. Wang, S. Kalytchuk, Y. Zhang, H. C. Shi, S. V. Kershaw, and A. L. Rogach, "Thickness-dependent full-color emission tunability in a flexible carbon dot ionogel," The Journal of Physical Chemistry Letters, vol. 5, no. 8, pp. 1412-1420, 2014.

[26] B. X. Feng, Z. Xu, J. Y. Wang, and L. G. Gai, "Water-soluble organic polymer/silica composite nanofilms with improved fluorescence quantum yield," Journal of Luminescence, vol. 211, pp. 347-354, 2019.

[27] Y. P. Sun, B. Zhou, Y. Lin et al., "Quantum- sized carbon dots for bright and colorful photoluminescence," Journal of the American Chemical Society, vol. 128, no. 24, pp. 7756-7757, 2006.

[28] J. Liang, Y. Jiao, M. Jaroniec, and S. Z. Qiao, "Sulfur and nitrogen dual-doped mesoporous graphene electrocatalyst for oxygen reduction with synergistically enhanced performance," Angewandte Chemie-International Edition, vol. 51, no. 46, pp. 11496-11500, 2012.

[29] C. H. Choi, M. W. Chung, S. H. Park, and S. I. Woo, "Additional doping of phosphorus and/or sulfur into nitrogendoped carbon for efficient oxygen reduction reaction in acidic 
media," Physical Chemistry Chemical Physics, vol. 15, no. 6, pp. 1802-1805, 2013.

[30] S. A. Wohlgemuth, R. J. White, M. G. Willinger, M. M. Titirici, and M. Antonietti, "A one-pot hydrothermal synthesis of sulfur and nitrogen doped carbon aerogels with enhanced electrocatalytic activity in the oxygen reduction reaction," Green Chemistry, vol. 14, no. 5, pp. 1515-1523, 2012.

[31] Y. J. Li, G. L. Wang, T. Wei, Z. J. Fan, and P. Yan, "Nitrogen and sulfur co-doped porous carbon nanosheets derived from willow catkin for supercapacitors," Nano Energy, vol. 19, pp. 165-175, 2015.

[32] Y. Q. You, H. R. Zhang, Y. L. Liu, and B. F. Lei, "Transparent sunlight conversion film based on carboxymethyl cellulose and carbon dots," Carbohydrate Polymers, vol. 151, pp. 245-250, 2016.

[33] X. Wang, L. Cao, S. T. Yang et al., "Bandgap-like strong fluorescence in functionalized carbon nanoparticles," Angewandte Chemie-International Edition, vol. 49, no. 31, pp. 5310-5314, 2010.

[34] S. T. Yang, X. Wang, H. F. Wang et al., "Carbon dots as nontoxic and high-performance fluorescence imaging agents," The Journal of Physical Chemistry C, vol. 113, no. 42, pp. 18110-18114, 2009.

[35] P. Anilkumar, X. Wang, L. Cao et al., "Toward quantitatively fluorescent carbon-based "quantum" dots," Nanoscale, vol. 3, no. 5, pp. 2023-2027, 2011.

[36] F. S. Niu, Y. H. Xu, J. X. Liu, Z. Q. Song, M. L. Liu, and J. Q. Liu, "Controllable electrochemical/electroanalytical approach to generate nitrogen-doped carbon quantum dots from varied amino acids: pinpointing the utmost quantum yield and the versatile photoluminescent and electrochemiluminescent applications," Electrochimica Acta, vol. 236, pp. 239-251, 2017.

[37] A. Gupta and C. K. Nandi, "PC12 live cell ultrasensitive neurotransmitter signaling using high quantum yield sulphur doped carbon dots and its extracellular $\mathrm{Ca}^{2+}$ ion dependence," Sensors and Actuators B: Chemical, vol. 245, pp. 137-145, 2017.

[38] C. Y. Yu, T. T. Xuan, Y. W. Chen et al., "Gadolinium-doped carbon dots with high quantum yield as an effective fluorescence and magnetic resonance bimodal imaging probe," Journal of Alloys and Compounds, vol. 688, pp. 611-619, 2016.

[39] R. Joseph, Lakovicz, Principles of Fluorescence Spectroscopy, Kluwer Academic/Plenum Publisher, New York, 2006.

[40] J. Lin, B. Huang, Y. F. Dai, J. C. Wei, and Y. W. Chen, "Chiral $\mathrm{ZnO}$ nanoparticles for detection of dopamine," Materials Science \& Engineering: C, vol. 93, pp. 739-745, 2018.

[41] C. L. Sun, C. T. Chang, H. H. Lee et al., "Microwave-assisted synthesis of a core-shell MWCNT/GONR heterostructure for the electrochemical detection of ascorbic acid, dopamine, and uric acid," ACS Nano, vol. 5, no. 10, pp. 7788-7795, 2011.

[42] W. Y. Chu, Q. Zhou, S. S. Li, W. Zhao, N. Li, and J. W. Zheng, "Oxidation and sensing of ascorbic acid and dopamine on selfassembled gold nanoparticles incorporated within polyaniline film," Applied Surface Science, vol. 353, pp. 425-432, 2015.

[43] M. H. Chen, Y. H. Zheng, J. W. Gao, C. Li, C. F. Yu, and Q. M. Wang, "Fluorometric determination of dopamine by using a terbium(III) inorganic-organic network," Microchimica Acta, vol. 184, no. 7, pp. 2275-2280, 2017.

[44] Y. Ma, A. Y. Chen, X. F. Xie et al., "Doping effect and fluorescence quenching mechanism of $\mathrm{N}$-doped graphene quantum dots in the detection of dopamine," Talanta, vol. 196, pp. 563-571, 2019.
[45] J. J. Zhao, L. M. Zhao, C. Q. Lan, and S. L. Zhao, "Graphene quantum dots as effective probes for label-free fluorescence detection of dopamine," Sensors and Actuators B: Chemical, vol. 223, pp. 246-251, 2016.

[46] J. J. Lv, S. S. Feng, Y. Ding et al., "A high-performance fluorescent probe for dopamine detection based on $\mathrm{g}-\mathrm{C}_{3} \mathrm{~N}_{4}$ nanofibers," Spectrochimica Acta Part A: Molecular and Biomolecular Spectroscopy, vol. 212, pp. 300-307, 2019.

[47] M. Yu, Y. Lu, and Z. J. Tan, "Fluorescence growth of selfpolymerized fluorescence polydopamine for ratiometric visual detection of DA," Talanta, vol. 168, pp. 16-22, 2017.

[48] S. Kulchat, W. Boonta, A. Todee, P. Sianglam, and W. Ngeontae, "A fluorescent sensor based on thioglycolic acid capped cadmium sulfide quantum dots for the determination of dopamine," Spectrochimica Acta Part A: Molecular and Biomolecular Spectroscopy, vol. 196, pp. 7-15, 2018. 


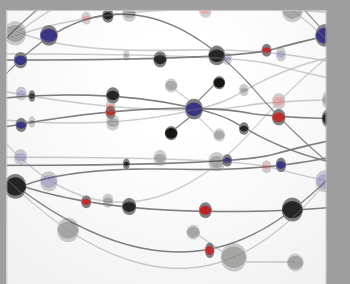

The Scientific World Journal
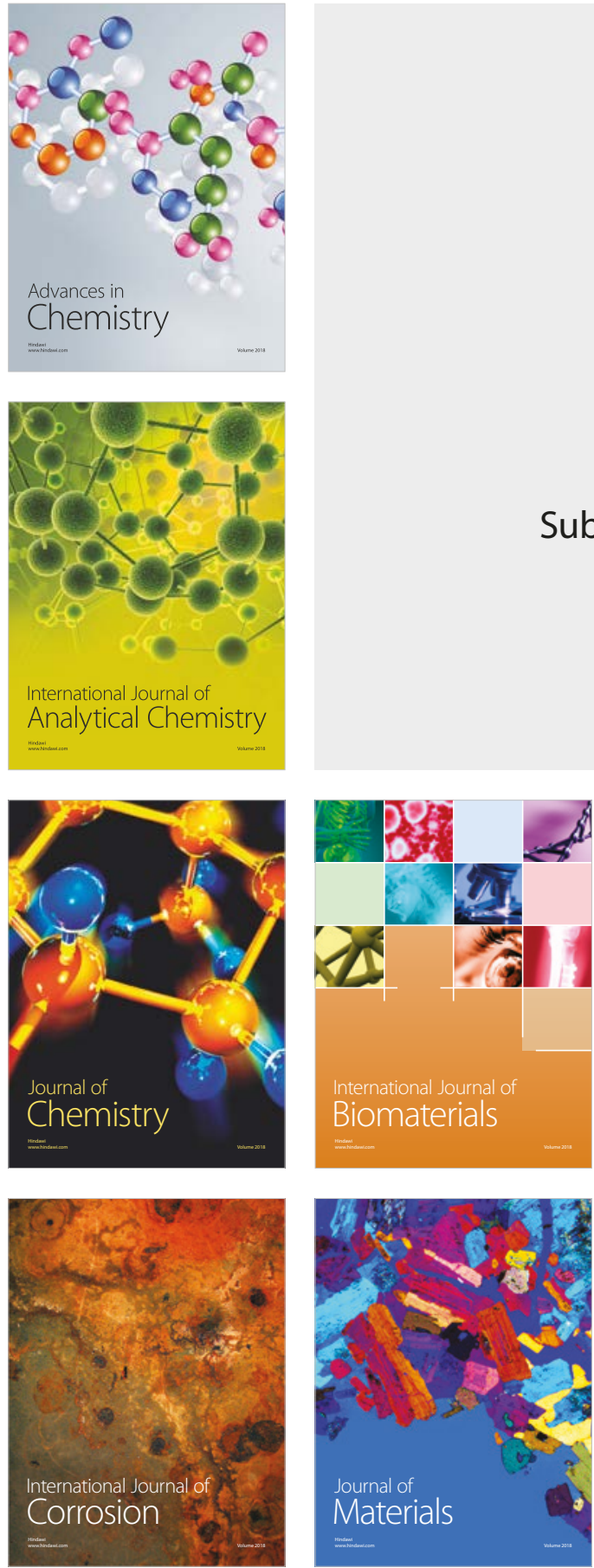

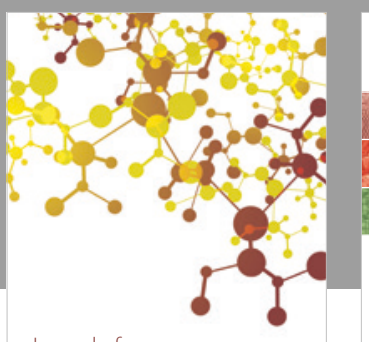

Journal of

Applied Chemistry
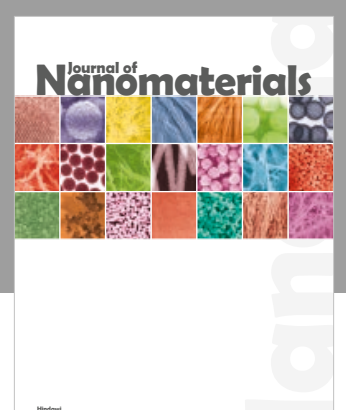

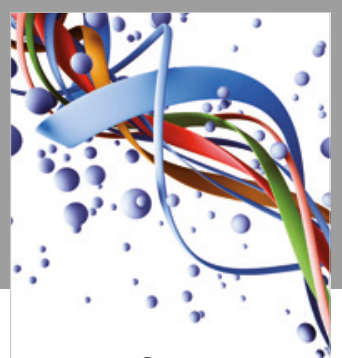

Scientifica

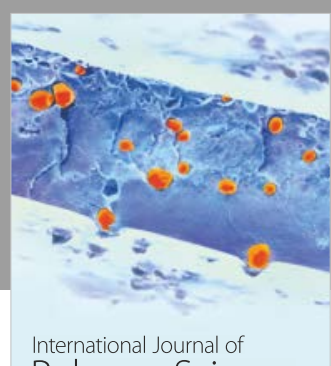

Polymer Science

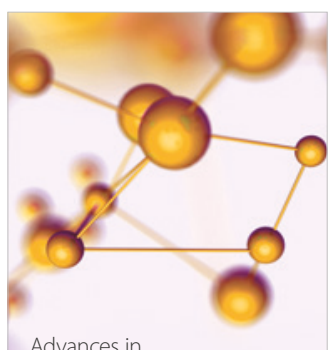

Physical Chemistry
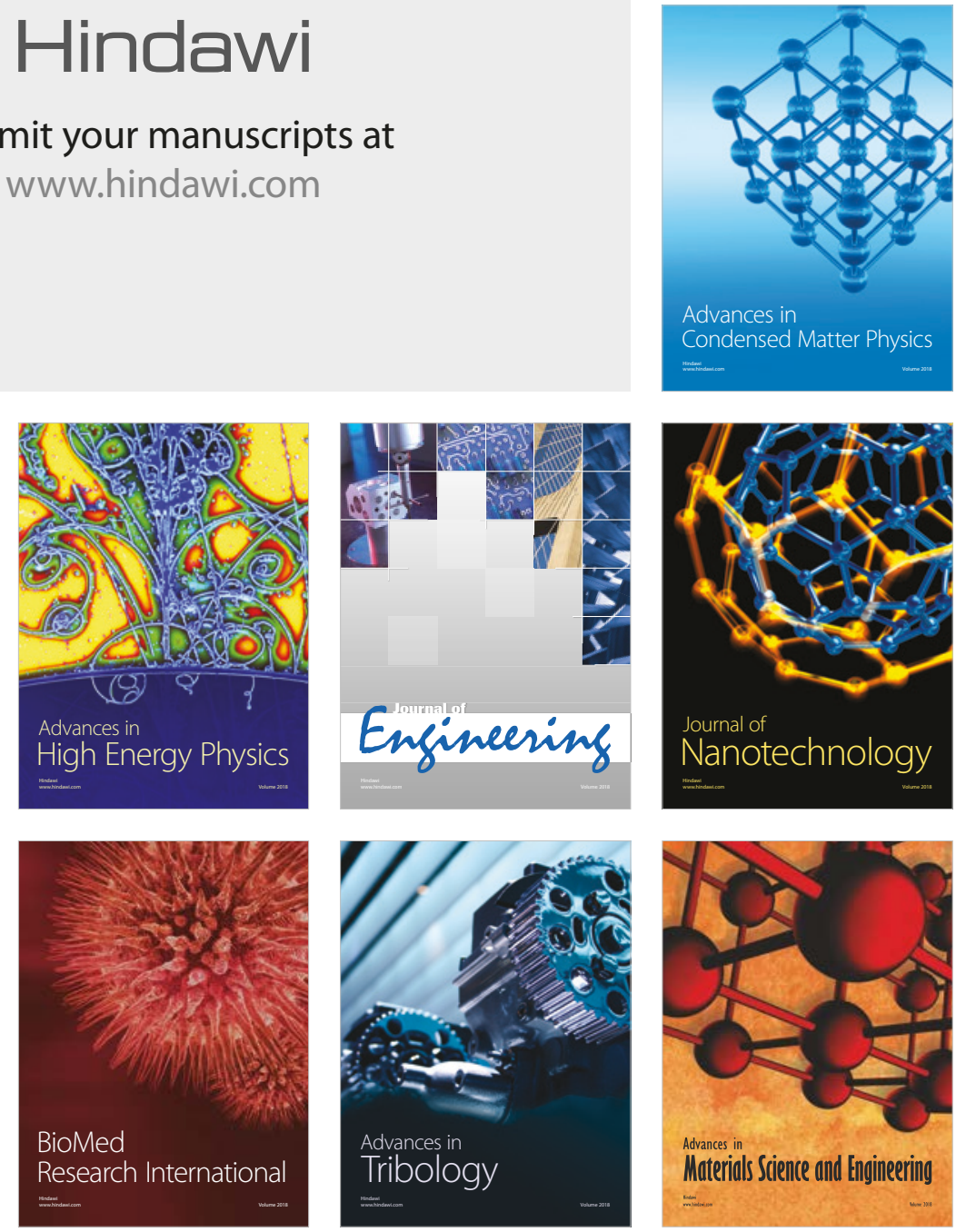